\title{
Boys, Be Independent! Conformity Development of Japanese Children in the Asch Experiment without Using Confederates
}

\author{
Kazuo Mori' ${ }^{1}$ Akina Ito-Koyama ${ }^{2}$, Miho Arai ${ }^{3}$, Aiko Hanayama ${ }^{4}$ \\ ${ }^{1}$ Department of Educational Psychology, Tokyo University of Agriculture and Technology, Tokyo, Japan \\ ${ }^{2}$ Faculty of Education, Shinshu University, Nagano, Japan \\ ${ }^{3}$ Department of Clinical Psychology, Tokyo Kasei University, Tokyo, Japan \\ ${ }^{4}$ Scientific Investigation Laboratory, Aomori Prefectural Police, Aomori, Japan \\ Email: kaz-mori@cc.tuat.ac.jp
}

Received 28 February 2014; revised 25 March 2014; accepted 21 April 2014

Copyright (C) 2014 by authors and Scientific Research Publishing Inc.

This work is licensed under the Creative Commons Attribution International License (CC BY).

http://creativecommons.org/licenses/by/4.0/

(c) (i) Open Access

\begin{abstract}
The Asch tasks were presented to 20 foursomes of Japanese $7^{\text {th }}$ graders $(10$ boy- and girl-foursomes) by means of a presentation trick so that one participant observed different stimuli than the other three. The response order was randomly assigned and the third responders observed different standard lines from the other three children. The results showed that the minority children who had observed different stimuli tended to conform to the majority. Combined with the previous results utilizing the same experimental procedure with two different age groupssix-year old children and undergraduates-a clear tendency was found for Japanese boys becoming more independent as they matured. Six-year-old Japanese boys conformed considerably, while male undergraduates seldom conformed, and the $7^{\text {th }}$ grade boys in the present study showed a moderate level of conformity. On the other hand, Japanese girls showed the same conformity frequencies regardless of age.
\end{abstract}

\section{Keywords}

Conformity, Gender Difference, Social Pressure, Junior High School Pupils

\section{Introduction}

Asch (1956) showed that a minority participant often conformed to the majority even when their choices seemed

How to cite this paper: Mori, K., Ito-Koyama, A., Arai, M., \& Hanayama, A. (2014). Boys, Be Independent! Conformity Development of Japanese Children in the Asch Experiment without Using Confederates. Psychology, 5, 617-623.

http://dx.doi.org/10.4236/psych.2014.57073 
incorrect. The majorities in those experiments consisted of confederates who had been instructed to respond incorrectly in several trials. The minority subject was a naïve participant. Asch's findings have been replicated using a number of different variations (see Bond \& Smith, 1996, for review).

However, there were few Asch experiments using child participants because it was difficult to find good child confederates. That was why Crutchfield (1955) developed an alternative experimental procedure designed to solve this problem of using confederates. In the Crutchfield procedure, each participant sat separately in a cubicle and received instructions that the small lights on the table in a cubicle would show the other participants' answers. Actually, the experimenter provided incorrect answers as if they were the actual responses of the other participants. In this way, the Crutchfield procedure avoided the problem of confederates and reproduced similar results on conformity as those from the Asch procedure.

Among the few studies that used child participants in conformity experiments was that of Costanzo and Shaw (1966), which used the Crutchfield procedure. They examined the conformity performance of children of three ages and adults and found that conformity was lowest for the youngest age group ( 7 - 9 year-olds), increased to the highest for the 11 - 13 year-olds, and decreased for the older age levels (15 - 17, and 19 - 21 year-olds). They did not find any statistical gender differences, although the conformity rates of girls were slightly higher at every age level.

Walker and Andrade (1996) conducted an Asch-type experiment with 110 Australian boys between the ages of 3 and 17. They selected child participants from each age group and trained them beforehand to act as experimental confederates. The experiments were conducted with four child participants, one naïve participant in the position of minority matched with a majority of three pre-trained child confederates. They found that the younger the children were, the more frequently they conformed. Eighty-five percent of the 3 - 5 year-olds children conformed, $42 \%$ of the 6 - 8 year-olds, 38\% of the $9-11$ year-olds, $9 \%$ of the $12-14$ year-olds, and none of the 15 - 17 year-olds conformed.

Whether children are subject to social pressure were further examined recently by other researchers. Corriveau and Harris (2010) asked 80 preschoolers of three to four year olds to choose the longest from three lines independently by themselves and with a group of adult informants. The preschoolers watched a movie in which three adults unanimously pointed a wrong line, and were asked to choose the longest. The results showed that about $44 \%$ of children conformed to the adult informants.

Haun and Tomasello (2011) investigated conformity of four-year-old children to their peers. In their experiment, foursomes of children were tested in a set of size judgment tasks. The crucial point of their experiment was that they did not use confederates but showed two different versions of tasks surreptitiously. Four children were tested together but only one of them were presented to tasks different from those presented to the other three. In this way, the performance of the minority child under social pressure was examined without using confederates. They found that minority children responded conform to the majority on 37.5\% (Experiment 1) and 31.5\% (Experiment 2) of trials.

Although these recent studies did show that children as young as three to five years old conform to majority, they did not add any new information about the development of conformity because they investigated only preschoolers. We need to compare the ratio of conformity with different age groups by utilizing the same experimental procedure. However, the procedures used both in Corriveau and Harris (2010) and Haun and Tomasello (2011) were not suitable for examining the conformity of older children. The trick invented by Haun and Tomasello worked for preschoolers, but it is dubious whether the same simple trick may work for older children or adult participants.

Mori and Arai (2010) invented a new experimental procedure for conducting conformity experiments called the fMORI-Asch procedure. Instead of using confederates, they utilized a presentation trick (the fMORI Technique; Mori, 2007) that could present two different visual stimuli without viewers' noticing the duality. They created PowerPoint slides showing the same stimulus sets that Asch (1956) had used. Then they made the standard lines that could be seen differently depending on the polarizing sunglasses that the participants wore. By having only one of the participants wear a different type of polarizing sunglasses, they reproduced the situation wherein the minority participant observed a standard line of a different length than the other viewers. With 26 Japanese undergraduate foursomes (10 male and 16 female foursomes), they found that the minority participants conformed to the majority. They also found a clear gender difference. Female participants conformed more often to the majority than males did. In fact, the male undergraduate participants seldom conformed.

Hanayama and Mori (2011) conducted an fMORI-Asch experiment with 24 foursomes of six-year old Japa- 
nese children (12 boy and 12 girl foursomes), and found that the minority children tended to conform to the majority. However, unlike Mori and Arai (2010) they found no gender differences; their 6 - 7 year-old boys showed similar levels of conformity frequency to girls of the same age.

These two studies clearly implied a possible developmental tendency for boys to become more independent as they mature. In contrast, girls may show a somewhat different developmental tendency. They may conform to their peers at roughly the same frequency regardless of their age. If this interpretation is correct, adolescents should show a moderate gender difference in conformity that would fit between the two experimental results. Therefore, we aimed to apply the same technique to Japanese junior high school pupils to examine whether their conformity patterns would confirm this interpretation.

It would have been desirable to conduct a series of experiments with new participants of three age levels. However, we conducted only one new experiment with $7^{\text {th }}$ grade pupils and re-used the data obtained from the two preceding studies with undergraduates and six-year-old children. This was mostly for the practical reason. One of the major demerits of the fMORI-Asch procedure is that it requires quadruple the number of participants because all the foursomes are genuine participants in that procedure. Consequently, in order to obtain the amount of data equivalent to the Asch experiments, four times more participants are needed. If the experiments had been conducted with three age levels it would have needed an unmanageably large number of participants.

\section{Method}

\subsection{Participants}

Eighty 7th-grade junior high school pupils (13 - 14 years old 40 boys and 40 girls) participated in same-sex groups of four. All the participants were from a municipal school in a middle-sized, rural city in Japan. The socio-economic status of the subjects' families varied within a narrow middle-class range. We made groups of children minimizing the within-group differences as much as possible. In each foursome, the response order was randomly assigned.

\subsection{Experimental Design}

A 2 (role: minority vs majority) $\times 2$ (gender: boys, girls) between-subjects factorial design was used. The dependent variable was the frequency of errors during the six critical tasks for each participant.

\subsection{Stimuli}

All the stimuli were exactly the same as those used in Mori and Arai (2010) and Hanayama and Mori (2011). Six of the nine stimulus sets were used for the critical tasks so that the minority viewer would observe different standard lines from the other three majority viewers (Figure 1).

\subsection{Apparatus}

The same experimental apparatus as used in Mori and Arai (2010) was set up in a vacant classroom at the junior high school. The stimuli were presented on PowerPoint slides with a personal computer (Apple iBook) and projected by an LCD projector (EPSON ELP-730) onto a rear screen made of plain ground glass $(80 \mathrm{~cm} \times 160 \mathrm{~cm})$. The rear screen was set about $1.4 \mathrm{~m}$ away from the projector. Four chairs were placed in a row about $2 \mathrm{~m}$ apart on the other side of the screen. A pair of polarizing sunglasses was placed on each chair before the participants entered the experiment room. Three identical pairs were used for the majority with one different type for the minority viewer. The minority participant's sunglasses were placed on the third chair.

\subsection{Procedure}

The participants were led by their schoolteacher into the experiment room and asked to take a seat in one of the four chairs. The seating order (answering order) had been randomly assigned beforehand. After they were seated with the sunglasses in hand, a female experimenter (the second author) gave the general instructions.

After all the instructions were given, the experimenter told participants to put on the sunglasses to protect their eyes from glare. Then, the experimenter presented the line judgment trials to the participants in the predetermined order. Each trial took approximately 30 seconds. 


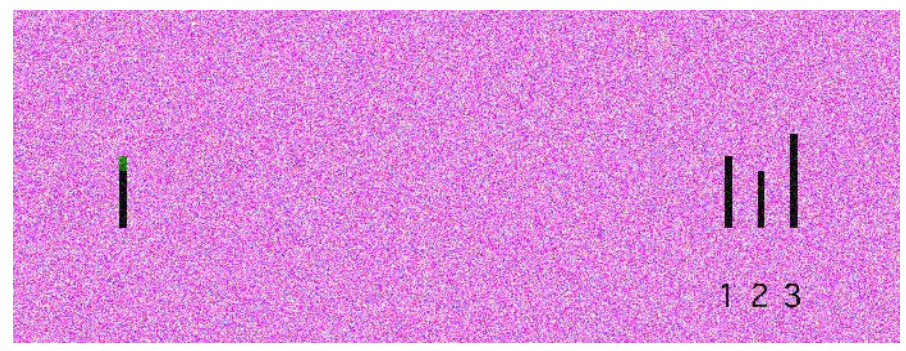

Figure 1. Task \#4 slide. The green part at the top of the standard line was either seen in black or hidden in the background depending on the type of polarizing sunglasses used.

\subsection{Post-Experimental Questionnaire}

Following the line judgment tasks, the participants filled in the questionnaire answering whether they had noticed any anomaly in the images or any visual illusions during the tasks.

\section{Results}

\subsection{Manipulation Check}

In the same manner as our previous studies, we examined whether the fMORI-Asch procedure successfully created majority and minority responders among genuine participants as intended for the sake of the experiment. The post-experimental questionnaire revealed that no participant noticed any anomaly during the tasks. The response patterns of the third responders and the other three responders showed that minorities performed differently from the others, revealing a clear minority-majority difference (Figure 2). Since there were no significant differences in error frequencies among the three responding orders of the majority participants $\left(\chi_{(2)}^{2}=2.65\right.$, ns.), we combined their data in the following data analyses.

\subsection{Conformity in the Minority Condition}

The minority participants made more errors than their peers, indicating that they had tended to conform to the majority. Unlike in the Asch experiments, all the participants were naïve in the fMORI-Asch experiments. Therefore, even those in the majority conditions made errors occasionally. Figure 3 shows the average number of errors of the participants for both the minority and majority conditions. The 2-way ANOVA on error frequencies of the experimental groups revealed that minority participants made significantly more errors than those in the majority conditions $\left(F_{(1,75)}=36.36, p<.01\right)$. We interpreted their highly frequent errors as occurrences of conformity to their majority peers. Female participants made more errors than males in the minority condition, whereas their error rates were similar in the majority condition. Therefore, we expected a significant interaction of gender and majority-minority conditions, but it did not reach a significant level $\left(F_{(1,75)}=1.54, p>.10\right)$. The main gender effect was not significant either $\left(F_{(1,75)}=1.67, p>.10\right)$.

\section{Discussion}

\subsection{Development of Conformity: An Integrated Analysis of Data from Our Previous Studies}

The results from the present study showed that $7^{\text {th }}$ graders in the minority condition tend to conform to their peers who formed a majority. Girls conformed more often than boys in the minority conditions, but the gender difference did not reach statistical significance. We compare these results with our previous studies with undergraduates and $1^{\text {st }}$ graders reported in Mori and Arai (2010) and Hanayama and Mori (2011), respectively.

Figure 4 shows the conformity rates of boys and girls in the present study along with those of the previous studies. In Hanayama and Mori (2011), the authors thought that the errors consisted of conformity responses and genuine perceptual errors. The genuine perceptual errors occurred irrespective of the experimental conditions. Therefore, the error rates observed in the majority conditions were the best estimates of the genuine perceptual error rates in the corresponding minority conditions. Then they calculated estimated conformity rates by 


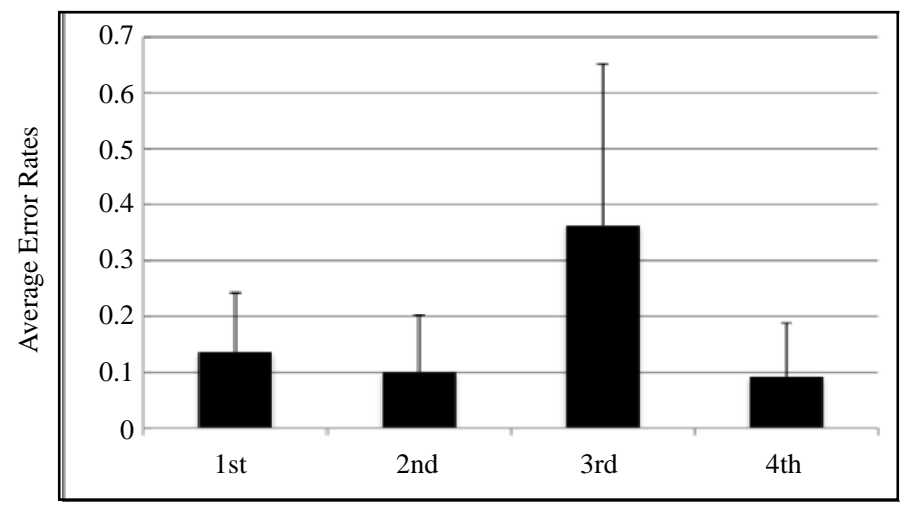

Response Oder

Figure 2. Average error rates in response order. The third responders were the minority participants. The vertical bars show standard deviations.

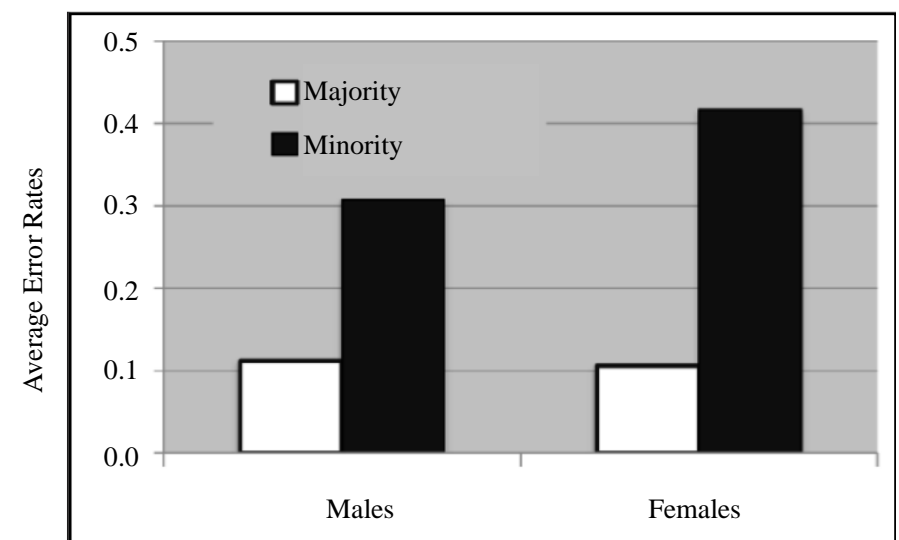

Figure 3. Average error rates in Majority Condition (in white) and in Minority Condition (in black).

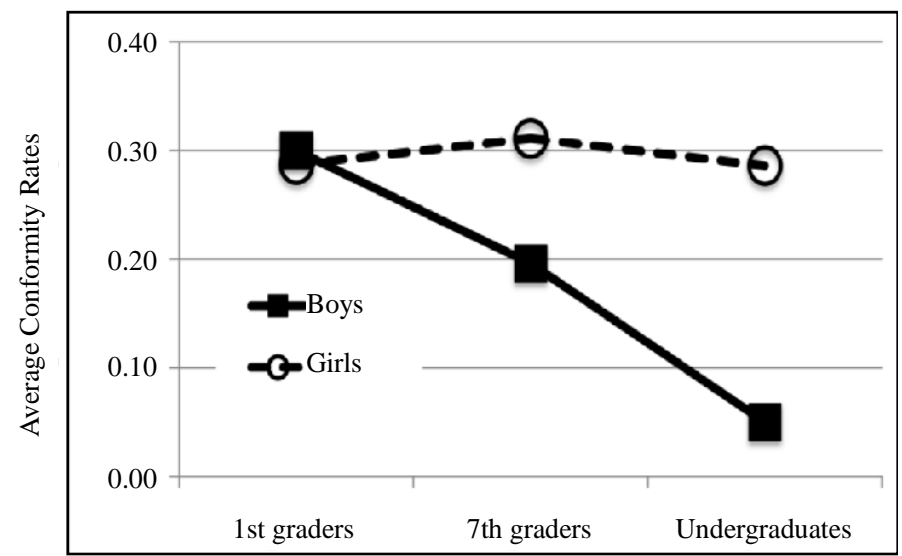

Figure 4. Developmental changes of conformity rates for boys and girls. The data of $1^{\text {st }}$ graders and undergraduates were taken from Hanayama and Mori (2011) and Mori and Arai (2010), respectively.

subtracting the error rates of the majority groups from those of the minority groups. The error rates for the $1^{\text {st }}$ grade boys and girls in the majority condition were 0.144 and 0.213 , respectively. We found error rates of around $10 \%$ in the present study, so we calculated equivalent conformity rates in the same way as Hanayama 
and Mori (2011) and used them in Figure 4. As for the undergraduate data of Mori and Arai (2010), we used the raw error rates in Figure 4, instead of the estimated ones, because the error rates were smaller than those of the male minority participants.

Figure 4 shows that the conformity rates of boys declined clearly whereas those of girls remained at a similar level regardless of age. We analyzed the error rates of these three age groups with a two-way ( 3 age levels $\mathrm{x} 2$ genders) ANOVA. The analyses revealed only the two main effects were significant $\left(F_{(2,64)}=6.59, p<.01\right.$, and $F_{(1,64)}=3.72, p<.10$, for age level and gender effects, respectively) but the interaction was not significant $\left(F_{(2,64)}\right.$ $=0.60$, ns). A separate one-way ANOVA revealed the gender difference was significant only for the undergraduates $\left(F_{(1,24)}=7.08, p<.05 ; F_{(1,22)}=.17\right.$ and $F_{(1,18)}=.68$, ns, for $1^{\text {st }}$ and $7^{\text {th }}$ graders, respectively).

\subsection{Development of Conformity: Comparisons to the Other Studies}

The Costanzo and Shaw (1966) study that used the Crutchfield procedure reported no gender difference in conformity development. In their study, both boys and girls showed similar developmental patterns following an inverted U shape with the highest conformity at the 11 - 13 year-old level. These results were completely different from our series of experiments in two ways. First, in our study conformity decreased as children matured. Secondly, although it did not reach a statistical significance, our study clearly showed a gender difference in conformity development. The conformity rates of girls were relatively stable regardless of age, whereas boys became more independent as they got older. This developmental tendency of boys was a replication of the results of Walker and Andrade (1996). However, since they did not use girl participants, they did not report anything about the conformity development of girls.

\subsection{Is the Gender Difference in Conformity Development Unique in Japanese?}

This is an interesting question but we simply lack crucial data to answer to it. It is safe to conclude here from the present data that the gender difference in conformity development was found in Japanese. The cultural differences between West and East have been studied in the contexts of "individualism versus collectivism" (Triandis, 1995) in social psychology. Asians are regarded as collectivists who view themselves primarily as parts of a collective entity. They are mainly motivated by the norms and duties imposed by the society they belong to. Therefore, they tend to conform to the group more frequently than individualists who are motivated by their own preferences, needs, and rights, giving priority to personal rather than to group goals.

However, only few experimental studies have clarified the differences between Western and Eastern cultures in conformity. In the thorough review of the literature in individualism and collectivism, Oyserman, Coon, and Kemmelmeier (2002) referred only to the Bond and Smith review (1996). We are simply lacking experimental data on gender difference in conformity development. It may be different from Japanese even other Asian children, such as Chinese or Korean. Therefore, we would like to urge researchers outside of Japan to conduct developmental studies in conformity behavior using the fMORI-Asch procedure with boys and girls in their cultures.

Why only Japanese boys grew up to be less conforming than girls is an interesting open question. It is assumed that there are stronger social expectations for boys to be independent than for girls in the Japanese tradition. There is a well-known anecdote in Japan about a former Professor at Hokkaido University, William Smith Clark [1826-1886], who left words at the time of his resignation to his Japanese students, "Boys, be ambitious!” School education in Japan has become gender-free after the World War II, but its hidden curriculum still requires “boys, be independent!”

\section{Conclusion}

We have conducted the Asch conformity experiments tasks with boy and girl foursomes of three different age groups; $1^{\text {st }}$ graders, $7^{\text {th }}$ graders, and undergraduates. The results showed a different developmental tendency in conformity for boys and girls. The Japanese boys tended to become less conforming as they grew. The $1^{\text {st }}$ grade Japanese boys conformed considerably (Hanayama \& Mori, 2011), while the male undergraduates seldom conformed (Mori \& Arai, 2010), and the $7^{\text {th }}$ grade boys in the present study showed a moderate level of conformity. On the other hand, Japanese girls showed the similar conformity frequencies throughout their development. We assumed that there are social expectations for Japanese boys to be independent. However, it is still an open question whether these gender differences in conformity development are unique to Japanese children or not, 
because we totally lack experimental studies on the gender differences in conformity development among various cultures.

\section{Acknowledgements}

This research was supported by a Grant-in-Aid from the Japanese Ministry of Education, Culture, Sports, Science, and Technology (KAKENHI Grant No.16653054) to KM while authors were at Shinshu University, Nagano, Japan. We wish to express our thanks to the principal and teachers of Oka Junior High School, Shiojiri, Nagano Prefecture, Japan, for the opportunity to conduct this experiment and to the pupils for participating in the experiments. We are indebted to Rebecca Ann Marck for her superb work in editing the English manuscript.

\section{References}

Asch, S. E. (1956). Studies of Independence and Conformity: I. A Minority of One against a Unanimous Majority. Psychological Monograph: General and Applied, 70, Whole No. 416.

Bond, R., \& Smith, P. B. (1996). Culture and Conformity: A Meta-Analysis of Studies Using Asch’s (1952b, 1956) Line Judgment Task. Psychological Bulletin, 119, 111-137. http://dx.doi.org/10.1037/0033-2909.119.1.111

Corriveau, K., \& Harris, P. L. (2010). Preschoolers (Sometimes) Defer to the Majority in Making Simple Perceptual Judgments. Developmental Psychology, 46, 437-445. http://dx.doi.org/10.1037/a0017553

Costanzo, P. R., \& Shaw, M. (1966). Conformity as a Function of Age Level. Child Development, 37, 967-975. http://dx.doi.org/10.2307/1126618

Crutchfield, R. S. (1955). Conformity and Character. American Psychologist, 10, 191-198. http://dx.doi.org/10.1037/h0040237

Hanayama, A., \& Mori, K. (2011). Conformity of Six-Year-Old Children in the Asch Experiment without Using Confederates. Psychology, 2, 661-664. http://dx.doi.org/10.4236/psych.2011.27100

Haun, D. B. M., \& Tomasello, M. (2011). Conformity to Peer Pressure in Preschool Children. Child Development, 82, 17591767. http://dx.doi.org/10.1111/j.1467-8624.2011.01666.x

Mori, K. (2007). Projecting Two Words with One Machine: A Method for Presenting Two Different Visual Stimuli Using Just One Projector without Viewer’ Noticing the Duality. Behavior Research Methods, 39, 811-815.

http://dx.doi.org/10.3758/BF03192973

Mori, K., \& Arai, M. (2010). No Need to Fake It: Reproduction of the Asch Experiment without Confederates. International Journal of Psychology, 45, 390-397. http://dx.doi.org/10.1080/00207591003774485

Oyserman, D., Coon, H. M., \& Kemmelmeier, M. (2002). Rethinking Individualism and Collectivism: Evaluation of Theoretical Assumptions and Meta-Analyses. Psychological bulletin, 128, 3-72. http://dx.doi.org/10.1037/0033-2909.128.1.3

Triandis, H. C. (1995). Individualism and Collectivism. New Directions in Social Psychology. Boulder, CO: Westview Press.

Walker, M. B., \& Andrade, M. G. (1996). Conformity in the Asch Task as a Function of Age. The Journal of Social Psychology, 136, 367-372. http://dx.doi.org/10.1080/00224545.1996.9714014 